\title{
Mobility Research of Six Pole Organizations
}

\author{
Mengxuan Xue ${ }^{1, a^{*}}$ and Yinwei Wang ${ }^{1, b}$ \\ ${ }^{1}$ College of Mechanical Engineering, Xijing University, Xi'an, China

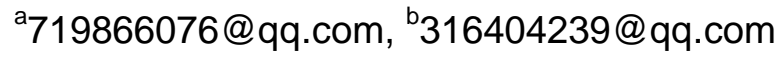

Keywords: Link motion gear; Six pole organizations; Crank; Six bar chains

\begin{abstract}
The link motion gear form is many and varied. The link motion gear may realize many kinds of path. The link motion gear obtains the quite widespread application in domains and so on aviation, light industry, metallurgy, robot, farm machinery. But so far, the link motion gear also has some elementary theory question not to be able to obtain the very good solution. The StePhenson six pole organization mobility question mainly includes the return route flaw, the crank existence question as well as the movement order question and so on. In this paper, six pole organization basic principle has been made the introduction; Six pole organization crank exists the condition has been carried on the analysis; Six pole organization movement order has been carried on the judgment. Distance curve section type corresponds which based on ( the S-SBM) return route, is carried on as the crank condition and the judgment method the research, the S-SBM crank question has been carried on the solution.
\end{abstract}

\section{Introduction}

Since most of planar linkage by unidirectional rotating motor drive, this request for comprehensive institutions must guarantee the existence of the crank, unlike four-bar linkage, multiple loop planar mechanism because the rotation of the different circuits have different sex, the crank existence of judgment is more difficult [1]. Six bar linkage crank existence conditions, including: all six bar linkage Watt, lotus root zero degree of S - SBM. Fig. 1 for stePhenson six bar mechanism motion principle diagram. But the Fig. 1 shows the existence conditions of the crank is incomplete; Due to the active parts relative to the frame even through a greater than 360 . The Angle of the produce oscillation, and didn't solve the problem of the institutions of the crank. Of S - SBM mobility problems, has not been a kind of system, suitable for computer automatic identification method, the research on this question should be comprehensive analysis of S - SBM and is of great significance.

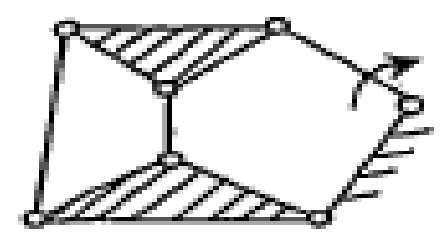

Figure 1. Motion principle diagram of the six bar mechanism

\section{Introduction of Six Bar Linkage}

In the need of engineering problems, always with its simplicity of four bar linkage being the better choice, but due to restrictions on the size, shape, and the transmission accuracy of often need to use more sophisticated institutions to meet the design requirements [2]. On the simplicity of six bar linkage is second only to four-bar linkage, its the advantage of simple low vice institutions at the same time, and can realize more complex kinematic requirements and good dynamic performance, but also has economic and durable than CAM mechanism, the advantages of higher speed than servo mechanism to provide. But because there are as many as six of six bar linkage loop, Wells and the 
rotation of the different circuits have different sex, so can choose in the synthesis of institutions through the defect in the expected movement range and there is no loop, movement sequence and crank problem difficulty sometimes is offset by the advantages of using six bar linkage, which hindered the use of it in the very people degree [3]. Stephenson six pole chain (shorthand for S - SBC), two three pole with four two pole connection, according to the different frame and active choice. Fig. 2 (a) is S - SBM - a diagram. Fig. 2 (b) is S - SBM - A diagram. Fig. 2 (c) is S - SBM - A diagram.

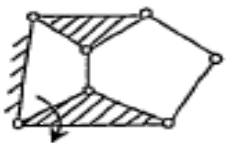

(a)

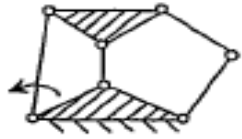

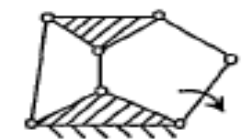

(b)

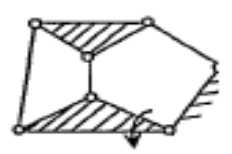

)

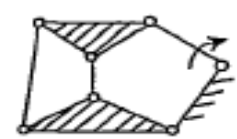

(c)

Figure 2. Diagram of S - SBM - A institutions

\section{Existence Condition of Six Bar Linkage Crank}

For single degree of freedom planar polycyclic linkage: if a multiple input values corresponding to the same circuit output value, indicates the circuit there are multiple branch, obviously, corresponding to the active parts of the circuit institutions can't rotate for the whole week, so there is no a crank [4].

Crank Existence Conditions and Judgment Method of S - SBM - A. Of S - SBM - A sufficient and necessary conditions for the active parts of the crank is: S - SBM - A BFBM for type 1; BFBM movement is not affected by double pole outside the group [5]. Clearly only by the loop identification standard 1 identified to satisfy these two conditions. If the circuit is composed of standard 1 identified, I then correspond to the loop I active thing that can be used as the crank, otherwise, the active piece not as a crank.

Crank Existence Conditions and Judgment Method of S - SBM - B. In S - SBM - B, a pair of G is the movement of the frame and vice connection active piece, this is a double pole outside group is a vice; Double pole group deputy to F, another deputy for E [6]. As active parts in double pole outside the group or the length of the frame components to 16, another member of length 1. For a B S from cicadas, according to the length of the two components in the external double pole, and seeds can be divided into two types:

Take Initiative to Group as Outer Double Pole Shorter Artifacts. Circuit corresponding distance curve segment E1E2 I to type 1. As shown in Fig. 3, E1 for loop point, E2 for each loop. If the initiative for the crank, you must satisfy: to track on the round a bit, to that point as the center of the circle, 15 for the radius of circle and E1E2 only one intersection point. Set the position of the components of the E1 and E2 GF GF1, GF2, respectively on E1E2 certainly exist some Ei, make the $\mathrm{FiEi}=15$, namely meet with Fi center, intersection Fi with E1E2 15 for the radius of circle [7]. So if the circle Fi with E1E2 always only one node (as shown in Fig. 3 (a)), it is a for loop I actively can be used as a song.Handle; Otherwise, if there are Fi with E1E2 node number greater than 1 (as shown in Fig. 3 (b)), it is not corresponding to the loop I actively for the crank.
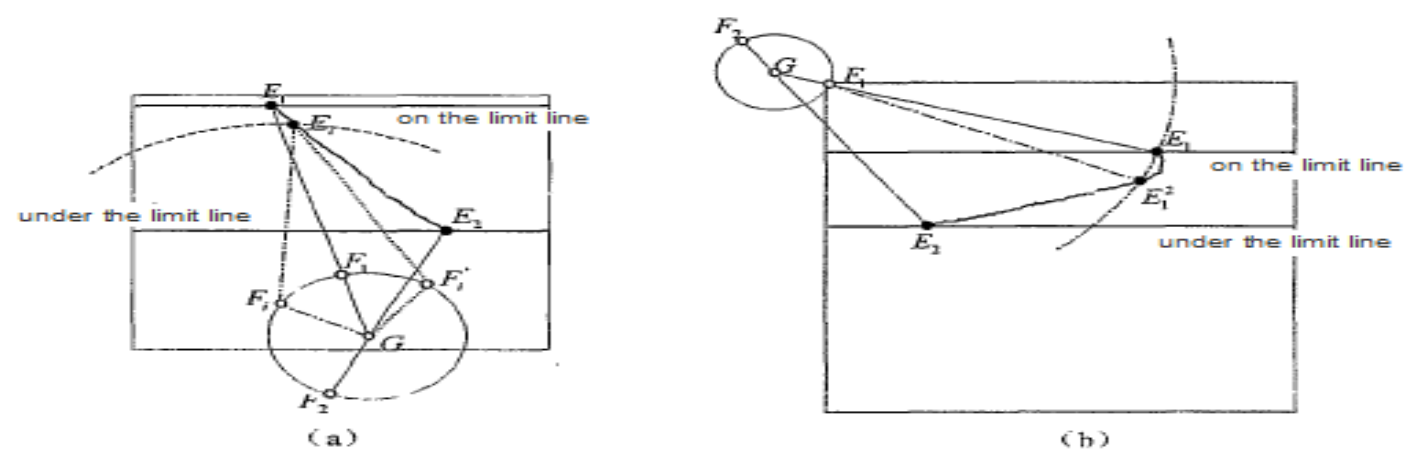

Figure 3. Judge figure of existence of the crank 
(2) Circuit corresponding distance curve segment E1E2 to type 2.For type 2 distance curve segment, according to its two endpoints E1 and E2, the properties of two kinds of circumstances: E1 and E2 are (or loop) circuit point on (as shown in Fig. 4 (a)). E1 for a loop on the point (loop), E2 loop points for () loop point on (as shown in Fig. 4(b) [8].

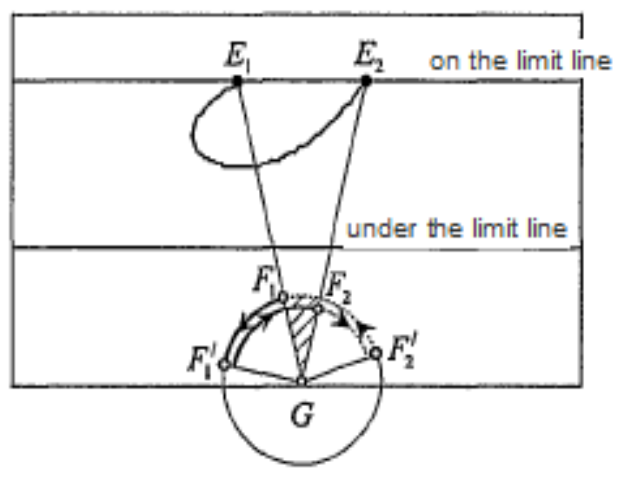

(a)

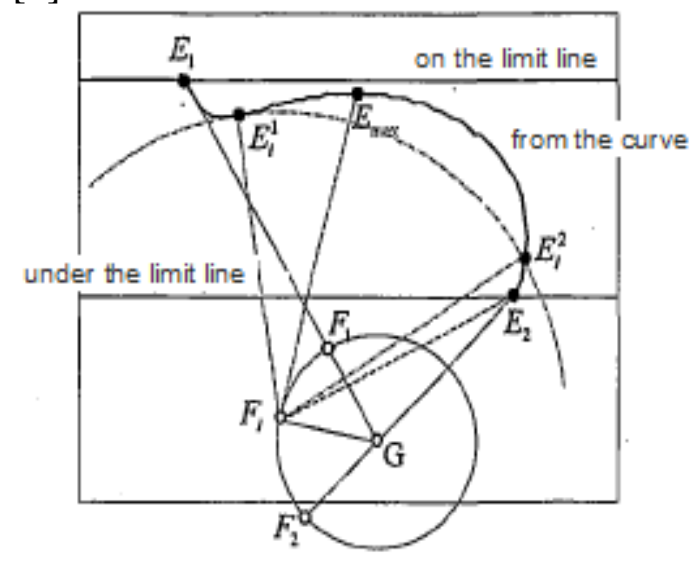

(b)

Figure 4. distance curve segment

In the light of the foregoing, for $\mathrm{S}$ a set active parts (SBM-B or rack) to double bar group in a relatively short period of components, if the loop i corresponds to the distance of the curve (above) exist, the value corresponding to the Loop S SBM-B active part certainly cannot be as crank.

Initiative to Double Bar Group Longer Component. (1) The distance corresponding to the $\mathrm{i}$ loop curve segments to Type 1E1E2. As shown in Fig. 6, the components of the Loop point corresponds to the E1E2 GF GF1 respectively, location GF2. Because the 16, 15 216-ls>15, >15 of radius and E1E2 certainly does not exist an intersection, thereby demonstrating that the initiative did not meet its part as the crank.(2) The Loop i corresponds to a distance curve (above) for type 2 of E1E2. The curve for type 2 (above) the loop, active part cannot be used as the Crank. Therefore, for S-SBM-B, if active (or rack) to double bar group in the longer components, active in always cannot be used as a crank [9].

\section{Judgment of Motion Sequence}

Organizations do not exist in the case of defects in the circuit, but also to determine if it meets the motion sequence, i.e. institutions during movement will in the prescribed order through the fine point. For a loop S-SBM if it is from the loop of the recognition criteria 1 identified, as long as the precise point corresponds to the virtual input $\mathrm{B}$, the values in the order given, institutions would not exist. Motion Sequence If it is from the Loop identified, their movement is more difficult to determine the order of this chapter, based on the body of the geostationary-bit-geostationary-bit-loop law to determine whether the institutions meet motion sequence.

The static configuration is the organization inherent nature, the organization may divide into two parts under the static configuration: Linear correlation and linear independence part, if takes initiative or some movement vice-achievement initiative by linear independence part some component, then cannot the drive rod group acting, have like the plane four pole organization blind spot, if the linear independence part for the non-driving part, is in the displacement in the static configurationally instantaneous organization the extreme value or the displacement function stagnation point position [10]. But therefore, the static configurationally existence and the position are the movement chain characteristic have nothing to do with the host moving parts choice, in a movement chain return route, which component the supervisor moving parts for, in its rate process, inevitably can't pass through in order this return route each static configuration. 


\section{Conclusions}

Any $\mathrm{S}$ a SBM regarding as is composed by BFBC and a double pole group, expands the SIDM return route analysis method to in a S SBC return route analysis, then a S SBM return route situation by $\mathrm{BFBC}$ return route and double pole group's configuration as well as BFBC and double pole group movement mutual function decision. Has realized S a SBM return route and the return route flaw automatic diagnosis. Distance curve section type corresponds which based on( the S-SBM) return route, obtained regarding each return route, judges each kind of S-SBM the main moving parts whether can take the crank the condition and the judgment method, for the first time systematic solution S-SBM crank question. For a solution to the problem of motion sequence S-SBM geostationary-bit-loop law for the first time, the precise point in integrated S-SBM the motion sequence, this method of having to conduct detailed campaign S-SBM analysis, and with the active part regardless of the selection.

\section{References}

[1] McCarthy J M, SohG S: Geometric Design of Linkages ( Springer Verlag, America 2010).

[2] Li Xuegang, Hang Yongqiang, Feng Liyan: A Sufficient and Necessary Condition for Existence of Double Crank of Planar Five Bar Linkage Studies , Vol. 24(2005).1.

[3] Ting K L, Wang Jun, Xue Changyu: Full Rotatability and Singularity of Six Bar and Geared Five Bar Likages, Vol 1(2010)No.2.

[4] Han Jianyou, Yang Tong, etc: Linkage modern integrated theory and method/parsing theory solution domain method and the software system (higher education press, China 2013).

[5] Yang Tong, Han Jianyou, Yin Lairong: Unified Synthesis Method Based on Solution Regions for Four Finitely Separated and Mixed "Point-Order" Position, Vol.46 (2011) No.11.

[6] Yang Tong, Han Jianyou: Research on Rigid-Body Guidance Mechanism Synthesis Through Four Mixed Positions, Vol .42 (2011) No.3.

[7] Luck K, Modler K H. Getriebetec: Analyse-Synthese-Optimierun (Akademie Verlag, Berlin 1990).

[8] Ting K L, Wang Jun, Xue Changyu: Full Rotatability and Singularity of Six-Bar and Geared Five-Bar Likages, Vol.2 (2010) No.1.

[9] Amir Rezaei, Alireza. Position: Jacobin and Workspace Analysis of a 3-PSP Spatial Parallelmanipulator, Vol.29 (2013) No.4.

[10] David E. Foster, Gordon R. Pennock: A Study on Kinematics and Dynamics of 3-DOF Parallel Machine, Vol.46 (2011) No.9. 